\title{
The role of 14,15-dihydroxyeicosatrienoic acid levels in inflammation and its relationship to lipoproteins
}

Tian Yang, Ran Peng, Yuan Guo, Li Shen, Shuiping Zhao and Danyan Xu*

\begin{abstract}
Background: 14,15-Epoxyeicosatrienoic acids (14,15-EETs) generated from arachidonic acid by cytochrome P450 epoxygenases have beneficial effects in certain cardiovascular diseases, and increased 14,15-EET levels protect the cardiovascular system. 14,15-EETs are rapidly hydrolyzed by soluble epoxide hydrolase (sEH) to the corresponding 14,15-dihydroxyeicosatrienoic acids (14,15-DHETs), which are generally less biologically active but more stable metabolite. A functionally relevant polymorphism of the CYP2J2 gene is independently associated with an increased risk of coronary heart disease (CHD), and the major CYP2J2 product is 14,15-EETs. 14,15-DHETs can be considered a relevant marker of CYP2J2 activity. Therefore, the aim of the present study was to evaluate the plasma 14,15-DHET levels to reflect the 14,15-EET levels in an indirectly way in patients with CHD, and to highlight the growing body of evidence that 14,15-EETs also play a role in anti-inflammatory and lipid-regulating effects in patients with CHD. This was achieved by investigating the relationship between 14,15-DHETs and high-sensitivity C-reactive protein (hs-CRP) and blood lipoproteins.
\end{abstract}

Methods: Samples of peripheral venous blood were drawn from 60 patients with CHD and 60 healthy controls. A 14,15-DHET enzyme-linked immunosorbent assay kit (14,15-DHET ELISA kit) was used to measure the plasma 14,15-DHET levels. Hs-CRP, total cholesterol, triglyceride, high-density lipoprotein cholesterol, and low-density lipoprotein-cholesterol levels were measured.

Results: 14,15 -DHET levels $(2.53 \pm 1.60 \mathrm{ng} / \mathrm{mL})$ were significantly higher in patients with CHD as compared with those of the healthy controls $(1.65 \pm 1.54 \mathrm{ng} / \mathrm{mL}, \mathrm{P}<0.05)$. There was a significant positive correlation between $14,15-\mathrm{DHET}$ and hs-CRP levels $(R=0.286, P=0.027)$. However, there was no significant correlation between 14,15-DHETs and blood lipoproteins (all, P > 0.05).

Conclusions: Increased plasma 14,15-DHET levels reflect the decreased of 14,15-EET levels in an indirectly way. Indicated that decreased plasma 14,15-EET levels might be involved in the inflammatory reaction process in atherosclerosis.

Keywords: Coronary heart disease, Atherosclerosis, 14,15-epoxyeicosatrienoic acids, 14,15-dihydroxyeicosatrienoic acids, Inflammation, Lipoproteins

\section{Introduction}

Over the past decade, it has become increasingly apparent that epoxyeicosatrienoic acids (EETs) have cardiovascular protective effects, including vasodilation, angiogenesis, decreasing platelet aggregation, and generally acting to maintain vascular homeostasis. More importantly, EETs have

\footnotetext{
* Correspondence: xudanyan02@sina.com

Department of Cardiology, Internet Medicine, The Second Xiangya Hospital, Central South University, Changsha 410011, Hunan, China
}

anti-inflammatory effects that play an important role in the prevention of coronary heart disease (CHD) [1-4]. EETs are hydrolyzed by soluble epoxide hydrolase (sEH) to the corresponding dihydroxyeicosatrienoic acids (DHETs); thus, it is expected that the inhibition of this enzyme enhances the beneficial cardiovascular properties of EETs [5]. Therefore, sEH inhibitors (sEHIs) have been rapidly developed and have been proven beneficial in cardiovascular diseases such as hypertension and CHD $[5,6]$.

\section{Biomed Central}


It is well known that inflammation plays a very important role in the development and prognosis of CHD. The initial findings of the anti-inflammatory properties of EETs described by Node et al. [7] that EETs inhibited the activation of nuclear factor kappa $\mathrm{B}(\mathrm{NF}-\mathrm{k} \mathrm{B})$, a key transcription factor involved in the expression of numerous pro-inflammatory genes. EETs were also found to inhibit the expression of vascular cell adhesion molecule-1 in human endothelial cells in response to tumor necrosis factor-alpha, interleukin-1 alpha, or lipopolysaccharide [7]. Some studies [8] have demonstrated that peroxisome proliferator-activated receptor gamma (PPAR $\gamma$ ) activation contributes to the anti-inflammatory effects of cytochrome P450 (CYP)-derived EETs. A number of studies [9-11] have also demonstrated that CYP-derived EETs inhibit cyclooxygenase 2-mediated inflammatory responses. Therefore, EETs might be an indicator reflecting the state of inflammation. But EETs are very unstable metabolites, it's rapidly hydrolyzed by $\mathrm{sEH}$ to the less biologically active but more stable metabolites DHETs. Spiecker et al. [12] demonstrated that a functionally relevant polymorphism of the CYP2J2 gene is independently associated with an increased risk of CHD, and the major CYP2J2 product is 14,15-EETs. 14,15DHETs can be considered a relevant marker of CYP2J2 activity. Therefore, in our study we chosen 14,15-DHETs to reflect the 14,15-EET levels in an indirectly way in patients with CHD.

From another point of view, high-sensitivity C-reactive protein (hs-CRP), an acute-phase reactive protein, is now recognized as a good indicator of inflammation and a pro-inflammatory atherogenic circulating marker that has been proven an independent cardiac risk predictor [13]. It has been shown that hs-CRP is of prognostic value in patients with acute coronary syndromes and plays an important role in the development of CHD [14]. However, the relationship between EETs and hs-CRP remains unclear.

Dyslipidemia also is an independent risk factor in the progress of CHD [15,16]. Low-density lipoprotein cholesterol (LDL-C) is atherogenic and represents a strong cardiovascular risk factor [17]. High-density lipoprotein cholesterol (HDL-C) mediates reverse cholesterol transport and exerts several atheroprotective effects [18]. Epidemiologic evidence has shown that low HDL-C is a strong and independent cardiovascular risk marker [19].

Some research has found that local adipose tissue inflammation and inflammatory lipid mediators, including EETs, may play important roles in regulating adipocyte function and lipid metabolism [20] and EETs can activate PPAR $\gamma$, which may play important roles in lipid metabolism [21]. However, the specific mechanism is still unknown; hence, we investigated the relationship between 14,15-DHETs and blood lipoproteins to determine whether they are related.
To date, there are no reports about the relationship between 14,15-EETs and hs-CRP and blood lipoprotein in patients with CHD. In order to explore the beneficial effects of EETs further, we studied the plasma 14,15DHET levels in patients with CHD, and investigated the relationship between 14,15-DHETs and hs-CRP as well as blood lipoproteins in these patients.

\section{Materials and methods \\ Subjects}

We recruited 120 people for this study. We enrolled 60 CHD patients ( 44 men and 16 women) ranging in age from 51 to 69 years old (average age $60.42 \pm 8.75$ years) in the study, which spanned September 2010 to December 2012 in our hospital. We recruited 60 age- and sexmatched healthy subjects as controls (42 men and 18 women, average age $61.09 \pm 8.87$ years).

The diagnosis of CHD was defined as having experienced symptoms of myocardial ischemia such as angina, and stenosis in at least one major epicardial coronary artery by coronary angiography. All participants were clinically stable and chest pain free at the time of their study visit. Patients with the following diseases and situations were excluded from the CHD group: left ventricular systolic dysfunction (ejection fraction $\leq 35 \%$ ), current use of insulin, active autoimmune disease, history of severe aortic stenosis, history of solid organ transplant or dialysis, or history of cancer within the previous five years. A detailed medical and medication history and fasting serum chemistry and cholesterol panel were obtained from the healthy volunteers; individuals with a history of cardiovascular disease or risk factors for coronary artery disease were excluded.

The Second Xiangya Hospital of Central South University Ethics Committee approved the study protocol. Blood was collected by venipuncture. Plasma was separated by centrifugation and stored at $-80^{\circ} \mathrm{C}$ pending analysis.

\section{Measurements and methods}

We collected 120 samples of peripheral venous blood, and then separated the plasma supernatant by centrifugation. An enzyme-linked immunosorbent assay (ELISA) was used to measure the plasma 14,15-DHET (14,15-DHET ELISA kit; Detroit R\&D Inc., Detroit, MI, USA) according to the manual. A specialist who was unaware of the subjects' assignations analyzed the plasma hs-CRP, total cholesterol (TC), triglyceride (TG), HDL-C, LDL-C, and biochemical indicators of liver and kidney function using a Hitachi 7170A analyzer (Hitachi, Tokyo, Japan).

\section{Statistical analysis}

All data were analyzed with SPSS 16.0. Continuous variables between groups were analyzed by an independent sample t-test. Correlations were tested by the Spearman 
rank correlation coefficient. Differences were considered significant if the null hypothesis could be rejected with $>95 \%$ confidence. P-values $<0.05$ (two-tailed) were considered to indicate statistical significance. The log-transformed hs-CRP and other values are presented as mean \pm standard error (mean $\pm \mathrm{SE}$ ).

\section{Results}

\section{Characteristics of study participants}

TG and LDL-C levels were higher in the CHD patients than in the control group $(\mathrm{P}<0.05)$, but the HDL-C levels of the CHD patients tended to be lower $(\mathrm{P}<0.05)$. No significant difference was observed for other indicators such as age, sex, body mass index, smoking status, $\mathrm{TC}$, fasting blood glucose, blood urea nitrogen, serum creatinine, alanine aminotransferase, and aspartate aminotransferase (all, $\mathrm{P}>0.05$; Table 1 ).

14,15-DHETs and hs-CRP levels between the two groups As shown in Table 2, the 14,15-DHET levels in the CHD group were significantly higher than that in the control group $(\mathrm{P}<0.05$; Table 2; Figure 1$)$, while hs-CRP levels were significantly higher in the CHD group $(\mathrm{P}<0.01$; Table 2; Figure 2).

\section{Correlation analysis of 14,15-DHETs and hs-CRP and blood lipoproteins}

We performed correlation analysis to elucidate the relationship between 14,15-DHETs and hs-CRP and blood lipoproteins. There was a significant positive correlation between 14,15-DHET and hs-CRP levels $(\mathrm{R}=0.286, \mathrm{P}=$ 0.027 ). However, there did not appear to be a significant correlation between 14,15-DHETs and blood lipoproteins such as TC, TG, LDL-C, and HDL-C $(\mathrm{P}>0.05$; Table 3; Figure 3).

\section{Discussion}

In the present study, we found higher serum 14,15-DHETs, hs-CRP, TG, and LDL-C concentrations in patients with $\mathrm{CHD}$ as compared to the control group, but HDL-C levels were lower in patients with CHD. There was also a significant positive correlation between 14,15-DHET and hs-CRP levels. However, there was no specific relationship between 14,15-DHETs and blood lipoproteins.

The results of the present study show that the 14,15-DHET levels were significantly higher in patients with CHD. The process of CHD is closely related with coronary endothelial injury and dysfunction, and myocardial ischemia. Endothelial cells play important roles in vasculogenesis and re-endothelialization after ischemic injury. One of the functions of EET that has been explored is the ability of EETs to prevent apoptosis and promote the growth of endothelial cells [22-24]. A potent inhibitor of apoptosis, 14,15-EETs can serve as an intracellular second
Table 1 Demographic and biochemical characteristics of study participants

\begin{tabular}{|c|c|c|c|}
\hline & $\begin{array}{c}\text { CHD patients } \\
\qquad n=60\end{array}$ & $\begin{array}{l}\text { Healthy controls } \\
\qquad n=60\end{array}$ & $\mathbf{P}$ \\
\hline Age (years) & $60.42 \pm 8.75$ & $61.09 \pm 8.87$ & NS \\
\hline Male/Female & $44 / 16$ & $42 / 18$ & NS \\
\hline $\operatorname{BMI}\left(\mathrm{kg} / \mathrm{m}^{2}\right)$ & $24.59 \pm 3.36$ & $24.63 \pm 2.91$ & NS \\
\hline Smoking cases (yes/no) & $38 / 22$ & $36 / 24$ & NS \\
\hline $\mathrm{TG}(\mathrm{mmol} / \mathrm{L})$ & $1.75 \pm 0.91$ & $1.33 \pm 0.68$ & $0.041^{a}$ \\
\hline $\mathrm{TC}(\mathrm{mmol} / \mathrm{L})$ & $4.48 \pm 1.01$ & $4.19 \pm 0.92$ & NS \\
\hline $\mathrm{HDL}-\mathrm{C}(\mathrm{mmol} / \mathrm{L})$ & $1.04 \pm 0.29$ & $1.20 \pm 0.28$ & $0.018^{a}$ \\
\hline LDL-C (mmol/L) & $2.78 \pm 1.10$ & $2.24 \pm 0.71$ & $0.036^{a}$ \\
\hline $\mathrm{FBS}(\mathrm{mmol} / \mathrm{L})$ & $6.49 \pm 3.06$ & $5.94 \pm 2.79$ & NS \\
\hline BUN (mmol/L) & $5.51 \pm 1.80$ & $5.67 \pm 1.40$ & NS \\
\hline Cr (umol/L) & $81.61 \pm 19.33$ & $78.85 \pm 16.87$ & NS \\
\hline $\mathrm{ALT}(\mathrm{mmol} / \mathrm{L})$ & $24.68 \pm 9.99$ & $20.58 \pm 8.21$ & NS \\
\hline AST (mmol/L) & $26.79 \pm 9.45$ & $24.24 \pm 7.44$ & NS \\
\hline $\begin{array}{l}\text { ACE inhibitor or } \\
\text { ARB }(\%)\end{array}$ & $36(60 \%)$ & $0(0 \%)$ & \\
\hline Beta-blocker (\%) & $49(82 \%)$ & $0(0 \%)$ & \\
\hline Statin (\%) & $60(100 \%)$ & $0(0 \%)$ & \\
\hline Aspirin (\%) & $60(100 \%)$ & $0(0 \%)$ & \\
\hline Clopidogrel (\%) & $15(25 \%)$ & $0(0 \%)$ & \\
\hline
\end{tabular}

${ }^{\mathrm{a}} \mathrm{P}<0.05$; CHD: Coronary heart disease; BMI: body mass index; TG: triglyceride TC: total cholesterol; HDL-C: high-density lipoprotein cholesterol; LDL-C: low-density lipoprotein cholesterol; FBS: fasting blood glucose; BUN: blood urea nitrogen; $\mathrm{Cr}$ : serum creatinine; ALT: alanine aminotransferase; AST: aspartate aminotransferase; NS: not significant.

messenger for epidermal growth factor in cells expressing epoxygenase activity, and promote endothelial cell growth [23]. Yan et al. [25] found that specific epoxidation of EET sites produces endogenous PPAR $\gamma$ agonists, increasing cell proliferators, which might affect angiogenesis and cardiac recovery after ischemic infarct and reperfusion. Xu et al. [26] also demonstrated that sEHIs can promote angiogenesis by activating the EET-PPAR $\gamma$ pathway, which in turn increases vascular endothelial growth factor and hypoxiainducible factor 1 alpha and triggers the migration and proliferation of endothelial progenitor cells. Numerous studies have suggested the beneficial effects of EETs on cardiac recovery following ischemia/reperfusion (I/R).

Table 2 14,15-DHETs and hs-CRP levels between the two groups

\begin{tabular}{lccc}
\hline & CHD patients & Healthy controls & $\mathbf{P}$ \\
& $\mathbf{n}=\mathbf{6 0}$ & $\mathbf{n}=\mathbf{6 0}$ & \\
\hline $14,15-\mathrm{DHETS}(\mathrm{ng} / \mathrm{ml})$ & $2.53 \pm 1.60$ & $1.65 \pm 1.54$ & $0.036^{\mathrm{a}}$ \\
$\mathrm{hs}-\mathrm{CRP}(\mathrm{mg} / \mathrm{L})$ & $2.92 \pm 1.20$ & $1.89 \pm 1.09$ & $0.001^{\mathrm{b}}$
\end{tabular}

${ }^{\mathrm{a}} \mathrm{P}<0.05 ;{ }^{\mathrm{b}} \mathrm{P}<0.01 ; 14,15$-DHETs: 14,15-dihydroxyeicosatrienoic acids; hs-CRP: high-sensitivity $\mathrm{C}$-reactive protein. 


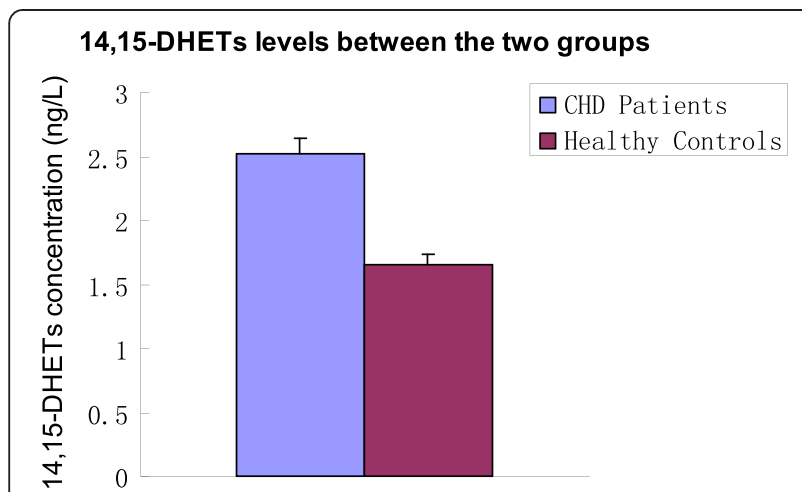

Figure 1 14,15-DHET levels between the two groups. CHD: Coronary heart disease; 14,15-DHETs: 14,15-dihydroxyeicosatrienoic acids.

Nithipatikom et al. [27] found that exogenous EETs produced a marked reduction in infarct size in dogs. Another study reported that the expression of CYP2J2 in cardiomyocytes led to improved functional recovery and reduced infarct size after ischemia [28]. EETs were reported to have aided in the preservation of mitochondrial integrity and membrane potential during I/R [29]. Similarly, EETs have been shown to release met-enkephalin, which binds $\delta$-opioid receptors to reduce infarct size after $I / R$ in rat heart [30]. EETs exert many other cardioprotective effects, including the reduction of myocardial stunning, myocardial infarct size, and inflammatory response; prevention of the onset of left ventricular hypertrophy and subsequent remodeling, which leads to heart failure; and reduction of the incidence of cardiac arrhythmias associated with heart failure [31]. Our findings are consistent with these results. But in Theken's [32] study, CHD patients had higher EETs and tended to have lower DHETs compared to healthy volunteers, and significantly lower apparent sEH metabolic activity in the presence of stable atherosclerotic cardiovascular disease. These results are differ from us, we can not rule out the possibility that the detected difference will achieve

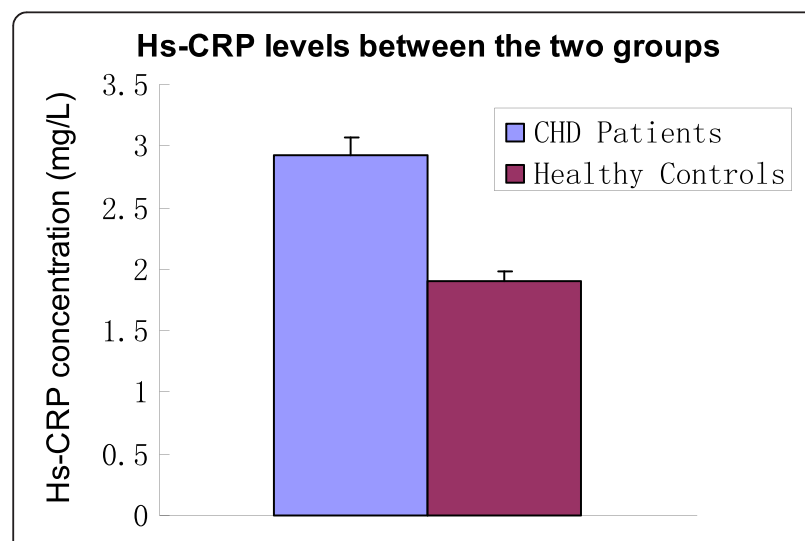

Figure 2 Hs-CRP levels between the two groups. CHD: Coronary heart disease; hs-CRP: high-sensitivity C-reactive protein.
Table 3 Related coefficients of 14,15-DHETs and hs-CRP, TG, TC, LDL-C, and HDL-C in patients with CHD

\begin{tabular}{cccccc}
\hline & hs-CRP & TG & TC & LDL-C & HDL-C \\
\hline 14,15-DHETs & 0.286 & 0.053 & 0.134 & 0.058 & -0.005 \\
& $(\mathrm{P}=0.027)^{\mathrm{a}}$ & $(\mathrm{P}=0.638)$ & $(\mathrm{P}=0.304)$ & $(\mathrm{P}=0.652)$ & $(\mathrm{P}=0.968)$
\end{tabular}

aP < 0.05; 14,15-DHETs: 14,15-dihydroxyeicosatrienoic acids; hs-CRP: highsensitivity C-reactive protein; TG: triglyceride; TC: total cholesterol; LDL-C: lowdensity lipoprotein cholesterol; HDL-C: high-density lipoprotein cholesterol.

statistical significance when future investigations study much larger patient groups. Even so, all the results lead up to the conclusion that EETs have a positive effect on the cardiovascular system.

We found that the plasma hs-CRP levels in patients with CHD were significantly higher than that in the control group. Inflammation is an important feature of atherosclerotic plaque [33,34]. As a vascular inflammatory marker, hs-CRP is closely related to cardiovascular disease. In patients with CHD, serum CRP levels gradually increase with the progress of the disease [35]. This is partially because CHD can cause ischemia and hypoxia, which leads to local tissue damage, myocardial infarction, myocardial fibrosis and necrosis, and neutrophil infiltration, thus stimulating the production of CRP. This clearly suggests that serum CRP levels are associated with the occurrence and development of CHD [35]. Schnell-Inderst et al. [13] found that the incidence of cardiovascular events was closely related to hs-CRP levels, and that hs-CRP can be used as an additional predictor in cardiovascular events.

We found a significant positive correlation between 14,15-DHET and hs-CRP levels in patients with CHD. This conclusion reflected that there might be a negative correlation between 14,15-EETs and hs-CRP in patients

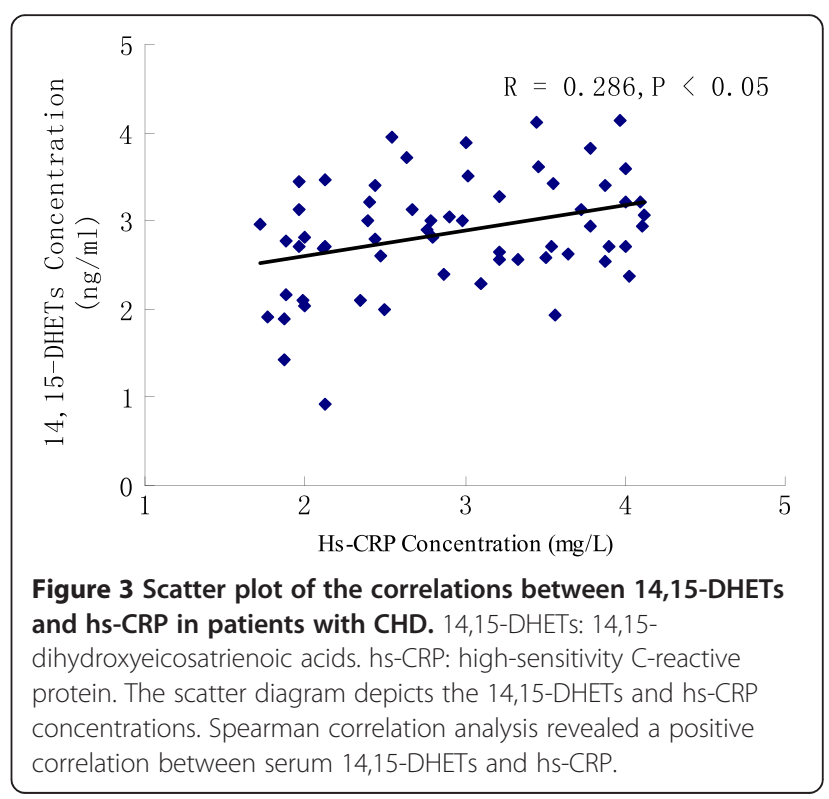


with CHD. Node et al. [36] found that EETs decreased cytokine-induced endothelial cell adhesion molecule expression and prevented leukocyte adhesion to the vascular wall by a mechanism involving the inhibition of the transcription factors NF- $\mathrm{kB}$ and inhibitor of kappa B kinase. The inhibitory effects of EETs were independent of their membrane-hyperpolarizing effects, suggesting that these molecules play an important nonvasodilatory role in vascular inflammation [36]. These findings and our results strongly suggest that EETs may be involved in the anti-atherosclerotic process by their inhibition of the inflammatory response. But in Schuck's [37] research, no associations were observed between biomarkers of CYP-mediated eicosanoid metabolism and hs-CRP, it suggest that CYP-derived eicosanoids may be important in the regulation of vascular, but not hepatic or systemic, inflammation in humans. These results are differ from us, we can not rule out the possibility that the detected difference will achieve statistical significance, or the medication used causes different results.

We found much evidence from epidemiologic, clinical, and laboratory data indicating that elevated TG levels are an independent risk factor for cardiovascular disease $[38,39]$. However, we found no significant correlation between 14,15-DHETs and TC, TG, LDL-C, and HDL-C. It is worth mentioning that some studies have demonstrated that sEHIs have anti-atherosclerotic effects, and that the anti-atherosclerotic effects are correlated with elevation in EET levels and associated with LDL-C reduction and HDL-C elevation, as well as attenuation of the expression of pro-inflammatory genes and proteins [40-42]. Zhang et al. [40] demonstrated that sEH inhibition could lower circulating cholesterol levels, which could also contribute to the attenuation of atherosclerosis. In contrast, many studies have demonstrated that lipoproteins play a key role in precipitating CHD [43]. Moreover, some studies have suggested that in view of its molecular structures, sEH is involved in cholesterol, fatty acid, and lipid metabolism [44-46]. It is known that EETs are potent endogenous PPAR $\alpha$ agonists, and as PPAR $\alpha$ activation can increase HDL-C by increasing the concentration of apolipoproteins A-I and A-II and by stimulating the reverse cholesterol transport pathway [47] it is expected to affect blood lipoproteins. However, we did not find a significant correlation between 14,15-DHETs and blood lipoproteins. Pritchard et al. [48] found that endothelial cells incubated in atherogenic LDL concentrations produced substantially greater quantities of EET species. Karara et al. [49] also found that the lipoprotein fraction with the highest EET concentration was LDL, followed by HDL and very low-density lipoprotein cholesterol. Thus far, no evidence shows that EETs and blood lipoproteins are not correlated. Therefore, we cannot rule out the possibility that the detected difference will achieve statistical significance when future investigations study much larger patient groups.

This study tested the relationship between 14,15-DHETs and hs-CRP and blood lipoproteins in patients with CHD. The in vivo cross-sectional design of the study presents several limitations. First, the levels of sEH and its enzymatic activity could be different between groups, 14,15-EET, 14,15-DHET, leukotoxin, and leukotoxin diol are potential biomarkers for assessing sEH activity in clinical trial subjects, our further studies are necessary to enroll these indicators to figure out the differences between two groups. It must also be noted that the we did not separated smoker and non-smoker, but there were no significant difference in the number of smokers between two groups, so the measured results are comparable. In addition, our analysis compared a well-treated population of patients with advanced cardiovascular disease to healthy individuals with no risk factors for cardiovascular disease. Thus, multiple potential confounding factors may have influenced the differences in 14,15-DHETs, hs-CRP and blood lipoprotein. We cannot determine whether the observed differences are due to the presence of atherosclerotic disease, or a consequence of drug therapy. Since the effects of these established therapies on circulating CYP-derived eicosanoid levels, and specifically sEH expression and metabolic activity, in humans are unknown, further studies are necessary to quantify these effects. And the number of patients enrolled in the study was relatively small, which might cause bias. Therefore, to expand the samples is what we need to do in our further researches.

\section{Conclusion}

In summary, the plasma 14,15-DHET levels in patients with CHD were significantly higher and were positive correlated with hs-CRP levels, suggesting that the decrease in 14,15-EET levels may be involved in the inflammatory reaction process in atherosclerosis. The correlation of 14,15-EETs and blood lipoproteins remains to be investigated.

\section{Consent}

Written informed consent was obtained from the patient for the publication of this report and any accompanying images.

\section{Competing interests}

The authors declare that they have no competing interests.

\section{Authors' contributions}

All the authors were involved in the design of this study. TY and LS substantially contributed to the design of the study, performing the experiment, analysis of data, and drafting the manuscript. RP carried out the concentration analysis. YG participated in sample selection. DYX and SPZ made contribution to design, analysis and revision of the manuscript. All the authors have read and approved the final version. 


\section{Acknowledgement}

This work was supported by National Nature Scientific Funding of China (No.81170190,81372117)

Received: 23 August 2013 Accepted: 18 October 2013

Published: 23 October 2013

\section{References}

1. Imig JD, Hammock BD: Soluble epoxide hydrolase as a therapeutic target for cardiovascular diseases. Nat Rev Drug Discov 2009, 8(10):794-805.

2. Michaelis UR, Fisslthaler B, et al: Cytochrome P450 2C9-derived epoxyeicosatrienoic acids induce angiogenesis via cross-talk with the epidermal growth factor receptor (EGFR). FASEB J 2003, 17(6):770-772.

3. Krötz F, Riexinger $T$, et al: Membrane-potential-dependent inhibition of platelet adhesion to endothelial cells by epoxyeicosatrienoic acids. Arterioscler Thromb Vasc Biol 2004, 24(3):595-600.

4. Fitzpatrick FA, Ennis MD, et al: Inhibition of cyclooxygenase activity and platelet aggregation by epoxyeicosatrienoic acids. Influence of stereochemistry. J Biol Chem 1986, 261:15334-15338.

5. Spector AA, Fang X, Snyder GD, Weintraub NL: Epoxyeicosatrienoic acids (EETs): metabolism and biochemical function. Prog Lipid Res 2004, 43:55-90.

6. Roman RJ: P-450 metabolites of arachidonic acid in the control of cardiovascular function. Physiol Rev 2002, 82(1):131-185.

7. Node K, Huo Y, et al: Anti-inflammatory properties of cytochrome P450 epoxygenase-derived eicosanoids. Science 1999, 285:1276-1279.

8. Liu $Y$, Zhang $Y$, et al: The anti-inflammatory effect of laminar flow: the role of PPAR-gamma, epoxyeicosatrienoic acids, and soluble epoxide hydrolase. Proc Natl Acad Sci U S A 2005, 102:16747-16752.

9. Kozak W, Aronoff DM, Boutaud O, Kozak A: 11,12-epoxyeicosatrienoic acid attenuates synthesis of prostaglandin E2 in rat monocytes stimulated with lipopolysaccharide. Exp Biol Med 2003, 228:786-794

10. Fang X, Moore SA, Stoll LL, Rich G, Kaduce TL, Weintraub NL, et al: 14,15-Epoxyeicosatrienoic acid inhibits prostaglandin E2 production in vascular smooth muscle cells. Am J Physiol 1998, 275:H2113-H2121.

11. Carroll MA, Balazy M, Margiotta P, Falck JR, McGiff JC: Renal vasodilator activity of 5,6-epoxyeicosatrienoic acid depends upon conversion by cyclooxygenase and release of prostaglandins. J Biol Chem 1993, 268:12260-12266.

12. Spiecker $M$, Darius $H$, Hankeln $T$, et al: Risk of coronary artery disease associated with polymorphism of the cytochrome P450 epoxygenase CYP2J2. Circulation 2004, 110(15):2132-2136.

13. Schnell-Inderst $P$, Schwarzer $R$, Göhler $A$, et al: Prognostic value, clinical effectiveness and cost-effectiveness of high sensitivity $C$-reactive protein as a marker in primary prevention of major cardiac events. Int J Technol Assess Health Care 2010, 26:30-39.

14. Sabatine MS, Morrow DA, et al: Prognostic significance of the Centers for Disease Control/American Heart Association high-sensitivity C-reactive protein cut points for cardiovascular and other outcomes in patients with stable coronary artery disease. Circulation 2007, 115(12):1528-1536.

15. Gordon T, Castelli WP, Hjortland MC, Kannel WB, Dawber TR: High density lipoprotein as a protective factor against coronary heart disease. The Framingham Study. Am J Med 1977, 62(5):707-714.

16. McQueen MJ, Hawken S, et al: Lipids, lipoproteins, and apolipoproteins as risk markers of myocardial infarction in 52 countries (the INTERHEART study): a case-control study. Lancet 2008, 372(9634):224-233.

17. Pöss J, Custodis F, Werner C, Weingärtner O, Böhm M, Laufs U: Cardiovascular disease and dyslipidemia: beyond LDL. Curr Pharm Des 2011, 17(9):861-870.

18. Barter P: HDL-C: role as a risk modifier. Atheroscler Supp/ 2011, 12(3):267-270

19. Trinidad R, Gómez J, García Mateo J, Magraner M, Bredy R: Independent association of LDL-C and non HDL-C with acute coronary syndrome in a Hispanic population. Bol Asoc Med P R 2011, 103(2):31-34.

20. Iyer A, Fairlie DP, Prins JB, Hammock BD, Brown L: Inflammatory lipid mediators in adipocyte function and obesity. Nat Rev Endocrinol 2010, 6(2):71-82.

21. Rosen ED, Sarraf P, Troy AE, Bradwin G, Moore K, Milstone DS, Spiegelman BM, Mortensen RM: PPAR gamma is required for the differentiation of adipose tissue in vivo and in vitro[J]. Mol Cell 1999, 4(4):611-617.

22. Chen JK, Capdevila J, Harris RC: Cytochrome P-450 epoxygenase metabolism of arachidonic acid inhibits apoptosis. Mol Cell Biol 2001, 21:6322-6331.
23. Chen J-K, Wang D-W, et al: Transfection of an active cytochrome P450 arachidonic acid epoxygenase indicates that 14,15-epoxyeicosatrienoic acid functions as an intracellular second messenger in response to epidermal growth factor. J Biol Chem 1999, 274:4764-4769.

24. Fleming I, Fisslthaler B, Michaelis UR, Kiss L, Popp R, Busse R: The coronary endothelium-derived hyperpolarizing factor (EDHF) stimulates multiple signalling pathways and proliferation in vascular cells. Pflugers Arch 2001 442:511-518.

25. Yan G, Chen S, You B, Sun J: Activation of sphingosine kinase-1 mediates induction of endothelial cell proliferation and angiogenesis by epoxyeicosatrienoic acids. Cardiovasc Res 2008, 78:308-314.

26. Xu DY, Davis BB, Wang ZH, Zhao SP, Wasti B, Liu ZL, Li N, Morisseau C, Chiamvimonvat N, Hammock BD: A potent soluble epoxide hydrolase inhibitor, t-AUCB, acts through PPARy to modulate the function of endothelial progenitor cells from patients with acute myocardial infarction. Int J Cardiol 2013, 167(4):1298-1304

27. Nithipatikom K, Moore JM, Isbell MA, Falck JR, Gross GJ: Epoxyeicosatrienoic acids in cardioprotection: Ischemic versus repefusion injury. Am J Physiol Heart Circ Physiol 2006, 291(2):H537-H542.

28. Seubert J, Yang B, et al: Enhanced postischemic functional recovery in CYP2J2 transgenic hearts involves mitochondrial ATP-sensitive $\mathrm{K}+$ channels and p42/p44 MAPK pathway. Circ Res 2004, 95(5):506-514.

29. Katragadda D, Batchu SN, Cho WJ, Chaudhary KR, Falck JR, Seubert JM: Epoxyeicosatrienoic acids limit damage to mitochondrial function following stress in cardiac cells. J Mol Cell Cardiol 2009, 46(6):867-875.

30. Gross GJ, Baker JE, Hsu A, Wu HE, Falck JR, Nithipatikom K: Evidence for a role of opioids in epoxyeicosatrienoic acid-induced cardioprotection in rat hearts. Am J Physiol Heart Circ Physiol 2010, 298(6):H2201-H2207.

31. Garrett J: Gross, kasem nithipatikom. Soluble epoxide hydrolase: a new target for cardioprotection. Curr Opin Investig Drugs 2009, 10(3):253-258.

32. Theken KN, Schuck RN, Edin ML, Tran B, Ellis K, Bass A, Lih FB, Tomer KB, Poloyac SM, Wu MC, Hinderliter AL, Zeldin DC, Stouffer GA, Lee CR: Evaluation of cytochrome P450-derived eicosanoids in humans with stable atherosclerotic cardiovascular disease. Atherosclerosis 2012, 222(2):530-536.

33. Biasucci LM, Aantamaria M, Liuzzo G: Inflammation, atherosclerosis and acute coronary syndromes. Minerva Caidioangiological 2002, 50:475-486.

34. Auer J, Berent R, Lassnig E, et al: C-reactive protein and coronary arterial disease. Jpn Heart J 2002, 43:607-619.

35. Schlager O, Exner M, Mlekusch W, Sabeti S, Amighi J, Dick P, Wagner O, Koppensteiner R, Minar E, Schillinger M: C-reactive protein predicts future cardiovascular events in patients with carotid stenosis. Stroke 2007, 38(4):1263-1268

36. Node $K$, Huo $Y$, Ruan $X$, et al: Anti-inflammatory properties of cytochrome P450 epoxygenase-derived eicosanoids. Science 1999, 285(5431):1276-1279.

37. Schuck RN, Theken KN, Edin ML, Caughey M, Bass A, Ellis K, Tran B, Steele S, Simmons BP, Lih FB, Tomer KB, Wu MC, Hinderliter AL, Stouffer GA, Zeldin DC, Lee CR: Cytochrome P450-derived eicosanoids and vascular dysfunction in coronary artery disease patients. Atherosclerosis 2013, 227(2):442-448.

38. Hokanson JE, Austin MA: Plasma triglyceride level is a risk factor for cardiovascular disease independent of high-density lipoprotein cholesterol level: a metaanalysis of population-based prospective studies. J Cardiovasc Risk 1996, 3:213.

39. Assmann $G$, Schulte $H$, Funke $H$, von Eckardstein A: The emergence of triglycerides as a significant independent risk factor in coronary artery disease. Eur Heart J 1998, 19(Suppl M):M8-M14.

40. Zhang LN, Vincelette J, Cheng Y, et al: Inhibition of soluble epoxide hydrolase attenuated atherosclerosis, abdominal aortic aneurysm formation, and dyslipidemia. Arterioscler Thromb Vasc Biol 2009, 29:1265-1270.

41. Ulu A, Davis BB, Tsai HJ, et al: Soluble epoxide hydrolase inhibitors reduce the development of atherosclerosis in apolipoprotein e-knockout mouse model. Cardiovasc Pharmacol 2008, 52:314-323.

42. Wang YX, Ulu A, Zhang LN, Hammock B: Soluble epoxide hydrolase in atherosclerosis. Curr Atheroscler Rep 2010, 12(3):174-183.

43. Cullen P: Evidence that triglycerides are an independent coronary heart disease risk factor. Am J Cardiol 2000, 86(9):943-949.

44. Enayetallah $A E$, Grant DF: Effects of human soluble epoxide hydrolase polymorphisms on isoprenoid phosphate hydrolysis. Biochem Biophys Res Commun 2006, 341:254-260.

45. Newman JW, Morisseau C, Harris TR, Hammock BD: The soluble epoxide hydrolase encoded by EPXH2 is a bifunctional enzyme with novel lipid phosphate phosphatase activity. Proc Natl Acad Sci USA 2003, 100:1558-1563. 
46. Harris TR, Aronov PA, Jones PD, Tanaka H, Arand M, Hammock BD: Identification of two epoxide hydrolases in Caenorhabditis elegans that metabolize mammalian lipid signaling molecules. Arch Biochem Biophys 2008, 472:139-149.

47. Nissen SE, Nicholls SJ, Wolski K, Howey DC, McErlean E, Wang MD, et al: Effects of a potent and selective PPAR-alpha agonist in patients with atherogenic dyslipidemia or hypercholesterolemia: two randomized controlled trials. JAMA 2007, 297:1362-1373.

48. Pritchard KA, Wong PYK, Stemerman MB: Atherogenic concentrations of Low-density lipoprotein enhance endothelial cell generation of epoxyeicosatrienoic acid products. Am J Pathol 1990, 136(6):1383-1392.

49. Karara A, Wei S, Spady D, Swift L, Capdevila JH, Falck JR: Arachidonic acid epoxygenase: structural characterization and quantification of epoxyeicosatrienoates in plasma. Biochem Biophys Res Commun 1992, 182(3):1320-1325.

doi:10.1186/1476-511X-12-151

Cite this article as: Yang et al:: The role of 14,15-dihydroxyeicosatrienoic acid levels in inflammation and its relationship to lipoproteins. Lipids in Health and Disease 2013 12:151.

\section{Submit your next manuscript to BioMed Central and take full advantage of:}

- Convenient online submission

- Thorough peer review

- No space constraints or color figure charges

- Immediate publication on acceptance

- Inclusion in PubMed, CAS, Scopus and Google Scholar

- Research which is freely available for redistribution 